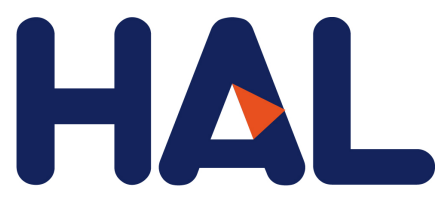

archives-ouvertes

\title{
Towards IP over LPWANs technologies: LoRaWAN, DASH7, NB-IoT
}

\author{
Wael Ayoub, Mohamad Mroue, Fabienne Nouvel, Abed Ellatif Samhat, \\ Jean-Christophe Prévotet
}

\section{To cite this version:}

Wael Ayoub, Mohamad Mroue, Fabienne Nouvel, Abed Ellatif Samhat, Jean-Christophe Prévotet. Towards IP over LPWANs technologies: LoRaWAN, DASH7, NB-IoT. 2018 Sixth International Conference on Digital Information, Networking, and Wireless Communications (DINWC), Apr 2018, Beirut, Lebanon. pp.43-47. hal-01991726

\section{HAL Id: hal-01991726 \\ https://hal.archives-ouvertes.fr/hal-01991726}

Submitted on 4 Feb 2019

HAL is a multi-disciplinary open access archive for the deposit and dissemination of scientific research documents, whether they are published or not. The documents may come from teaching and research institutions in France or abroad, or from public or private research centers.
L'archive ouverte pluridisciplinaire HAL, est destinée au dépôt et à la diffusion de documents scientifiques de niveau recherche, publiés ou non, émanant des établissements d'enseignement et de recherche français ou étrangers, des laboratoires publics ou privés. 


\title{
Towards IP over LPWANs technologies: LoRaWAN, DASH7, NB-IoT
}

\author{
Wael Ayoub* ${ }^{* \dagger}$, Mohamad Mroue ${ }^{\dagger}$, Fabienne Nouvel* ${ }^{*}$ Abed Ellatif Samhat ${ }^{\dagger}$, and Jean-christophe Prévotet ${ }^{*}$ \\ *Institut National des Sciences Appliquées de Rennes - IETR-INSA, Rennes, France. \\ $\dagger$ Faculty of Engineering - CRSI, Lebanese University, Hadath Campus, Hadath, Lebanon

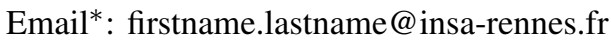 \\ Email ${ }^{\dagger}$ : samhat@ul.edu.lb, mkmroue@gmail.com
}

\begin{abstract}
In this paper, we discuss a set of solutions that are proposed in order to run IPv6 over IoT and we investigate its applicability over the three Low Power Wide Area Networks (LPWANs) technologies: LoRaWAN, DASH7, NB-IoT. LPWANs are wireless technologies that are used to connect things to the Internet. These technologies are characterized by their large coverage area, long battery operation, low bandwidth, small frame payload size, and the use of asymmetric and non-synchronized communication. Based on this investigation, we highlight the schemes that can be adopted for IP-based LPWANs technologies.

Index Terms-IoT communication, LPWAN, LoRaWAN, DASH7, NB-IoT, IPv6, adaptation layer, IP compression.
\end{abstract}

\section{INTRODUCTION}

Gartner [1] predicts that there will be more than 20.8 billions of smart things that will be connected to Internet by 2020 , whereas the worldwide number of devices was 6.4 billions in 2016. This growth will continue to increase exponentially over the next decade. Yet, IPv4 is unable to meet this growth even with network address translation (NAT) and private address space. The limitation of IPv4 address space made the transition to IPv6 unavoidable. Using IPv6 leads to expand the address space to $2^{128}$ which is equivalent to 4000 address for every person on the planet.

Indeed, it is commonly recognized that the core network will be based on IPv6 [2] for the next-generation Internet. Also, Google figures an exponential curve for the adoption of IPv6 that is doubling every 6 months. IPv6 traffic represents over $22 \%$ of Google Internet traffic [3]. Recently, a lot of researchers are proposing to adopt IPv6 with heterogeneous networks including the Low Power energy technologies with resource constraints, e.g.: IoT6 [4].

The Internet protocol is a mandatory for Internet connectivity [5]. IPv6 provides for IoT many advantages and its achievement is just a matter of time [6], but it is impossible to apply it directly to LPWANs technologies even with the use of fragmentation. The overhead of IPv6 is not compatible with LPWANs technologies, because 32 bytes are reserved for the source and destination addresses, 16 bytes for each. For that, several solutions to adopt IPv6 in IoT were proposed and only successful ones were standardized. In this paper, we investigate those standardized solutions and we study their applicability with our chosen LPWANs technologies.

First, we review the previous and current solutions created to benefit from IPv6 features on constrained networks. Those solutions provide a form to run IPv6 over IoT technologies and the successful ones were standardized. From IoT technologies, we focus in this paper on LPWANs technologies. In the following, we will present these solutions and analyze their applicability with the three LPWAN technologies: LoRaWAN, DASH7, and NB-IoT.

The rest of this paper is organized as follows. First, section II provides an overview and describes the architectures of the three LPWAN standards, LoRaWAN, DASH7, and NB-IoT. Also, we present their specifications and related challenges. Then, in Section III, we present the standardized protocols to run IPv6 over IoT and we describe the encountered limitations. Finally, section IV provides a conclusion and discusses the future works.

\section{LPWAN TECHNOLOGIES}

LPWANs are a new type of wireless communication technologies that are designed to support exchange of data with low bandwidth for long range on battery powered devices. This type of technologies is proposed to address the IoT needs and to support machine to machine (M2M) networks. Its aim is to operate with low cost and minimum power consumption. LPWANs represent a new phenomenal model in communication. This phenomena complements between cellular networks and the short wireless technologies that address the diversity of IoT applications. These technologies are designed to offer a set of features including wide-area and massive scale connectivity for low data rate, low energy consumption, and low cost devices that are not provided by existing legacy wireless technologies.

\section{A. Architecture}

In the basics, the three LPWANs share similar system architecture as shown in Fig. 1 but with different terminologies. The system architecture is formed by:

- End Device/Tags/User Equipment (ED): is the client device that sends or receives data. It usually refers to sensors, detectors, actuators and where sensing and controlling take place. Their number can be high within a gateway.

- Gateway/ENodeB (GW): also called modem, access point, or base station. It gets/pushes data between the core network and ED. It communicates via IP with the core network.

- Network Server/Cloud server/Serving-GW (NS): is the most intelligent part of the network. It is responsible for monitoring the $\mathrm{GW}$ and $\mathrm{ED}$, aggregating the incoming data, and Routing/forwarding incoming messages to the corresponding application server. 
TABLE I

LORAWAN PROTOCOL SPECIFICATIONS

\begin{tabular}{|l|c|}
\hline Specification & LoRaWAN Technology Support \\
\hline Standard & LoRa Alliance \\
\hline Operational & UnLicensed ISM band \\
Frequences & $868,915 \mathrm{MHz}$ \\
\hline Data Rate (kbps) & $0.3-50$ (Europe) \\
& $0.9-100$ (US) \\
\hline Identity header size & 4 bytes \\
\hline Payload size & $51-222$ bytes \\
\hline Addressing & UL: Broadcast \\
& DL: Unicast \\
\hline Topology & Star \\
\hline
\end{tabular}

- Join Server/Assign server/Home subscriber Server: is responsible of joining an ED to network and controlling the ED authentication.

- Application Server/Cloud Application/Packet Data Node Gateway (AS): refers to a program or code running on the user side to communicate with $\mathrm{ED}$ in order to send/receive data.

Architecture of: LoRaWAN, DASH7, and NB-IoT

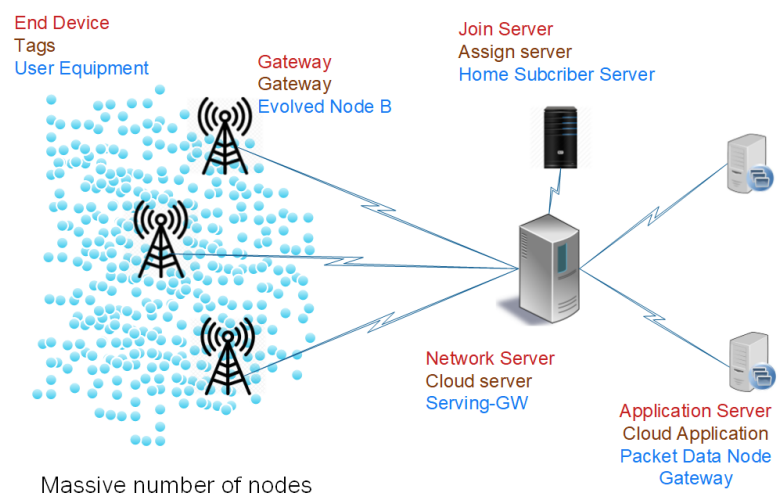

Fig. 1. General System Architecture

\section{B. LoRaWAN}

LoRaWAN is an open standard architecture developed by LoRa Alliance [7] to provide a medium access control mechanism, and enable ED to communicate with one or more gateways. LoRa is a physical layer technology that enables long range, low data rate, and low power wireless communication. It is an unlicensed technology that modulates the signals in the Sub GHz ISM band using a spread spectrum technique [8]. It is commercialized by Semtech [9]. LoRa can also be used in M2M communications between nodes. Table I shows the specifications of the LoRa protocol. LoRaWAN is a link layer protocol above the LoRa physical layer protocol.

\section{DASH7}

The DASH7 Alliance (D7A) [10] is an open source active RFID standard for Wireless Sensor and Actuator Network Protocol. D7A complies with the ISO/IEC 18000-7 Which is an open standard for the license free $433 \mathrm{MHz}$ ISM band airinterface for wireless communication. A full OSI stack (7 OSI layers) known as D7A protocol (D7AP) is specified [11]. It provides a long range (up to $2 \mathrm{Km}$ ), and low latency with multi-year battery life to connect moving objects. Table II shows the specifications of D7AP technology.

TABLE II

D7A PRotocol SPECIFICATIONS

\begin{tabular}{|l|c|}
\hline Specification & DASH7 Technology Support \\
\hline Standard & ISO/IEC 18000-7 \\
\hline $\begin{array}{l}\text { Operational } \\
\text { Frequences }\end{array}$ & UnLicensed ISM band \\
\hline Data Rate (kbps) & $\begin{array}{c}13,56,55,200 \\
(16,8,4 \text { channels) }\end{array}$ \\
\hline Identity header size & $2-8$ bytes \\
\hline Payload size & 256 bytes (Max) \\
\hline Addressing & Unicast - Broadcast \\
& Multicast - Anycast \\
\hline Topology & Star \\
\hline
\end{tabular}

\section{NB-IoT}

Narrow Band Internet of Things (NB-IoT) is a part of release 13 [12]. It was setup by 3GPP in Cellular system in support for ultra-low complexity and low throughput Internet of Things (CIoT). It defines a new radio access technology that can be integrated into the LTE standard. NB-IoT is built from existing LTE functions but many features have been removed to keep this standard as simple as possible in order to reduce device cost and minimize battery consumption. This optimization includes removing handover, carrier aggregation, measurements to monitor the channel quality, and dual connectivity. NB-IoT operates on the same licensed frequencies used by LTE, and employs QPSK modulation. Table III shows the specifications of NB-IoT. NB-IoT is designed to fit in 200 $\mathrm{kHz}$ system bandwidth used by both uplink and downlink. This enables NB-IoT to gain the feature of deployment in the GSM carrier as standalone, and in LTE as in-Band or Guard-Band.

TABLE III

NB-IOT PROTOCOL SPECIFICATIONS

\begin{tabular}{|l|c|}
\hline Specification & NB-IoT Technology Support \\
\hline Standard & 3gpp (release 2015) \\
\hline $\begin{array}{l}\text { Operational } \\
\text { Frequences }\end{array}$ & Licensed \\
\hline Data Rate (kbps) & Same as LTE bands \\
\hline Identity header size & As LTE \\
\hline Payload size & UL: 125 bytes \\
& DL: 85 bytes \\
\hline Addressing & UL: Unicast \\
\hline Topology & DL: Unicast and Broadcast \\
\hline \multicolumn{2}{|c|}{ UL: data frame transmitted from ED to the GW } \\
DL: data frame transmitted from GW to the ED.
\end{tabular}

\section{E. Challenges}

The LPWANs technologies are large-scale constrained networks with the following characteristics:

- Small payload size as low as 51 bytes in LoRaWAN.

- Limitation on the number of messages per day according to adopted local regulations. 
- Very low bandwidth, between $50 \mathrm{bit} / \mathrm{s}$ and $250 \mathrm{kbit} / \mathrm{s}$.

- High packet loss, due to collisions and bad transmission (e.g., devices in LoRaWAN use ALOHA mechanism to transmit messages).

- Variable MTU for a link depending on the used L2 modulation.

- Asymmetric communication (usually UL) and in some cases unidirectional links.

- Massive number of nodes per one gateway.

- Different modulations and radio channels.

- Most of the time ED is in sleep mode to preserve energy.

\section{ANALYSIS OF THE EXISTING IOT STANDARDS}

IPv6 offers several features and arguments that demonstrate its success in the future of IoT. A brief listing of the main features of IPv6 is given in the following:

- Scalability: Address space of $2^{128}$ equivalent to $3.4 \times 10^{38}$ address. This allows assigning more than IP to each ED.

- Overcomes the NAT barriers that are found in IPv4.

- Multiple IP addresses per device: This enables sharing a common device between infrastructures.

- Group operation and Multicast: This enables a device to join a group, and a single operation is performed on the whole group.

- Mobility and security support better than IPv4.

- Stateless Address Auto-configuration (SLAAC): It allows a massive number of IoT devices to join the network and connect to Internet without using DHCP server.

- Availability of a set of complementary standards and solutions for the IoT needs: e.g. 6LoWPAN, 6TiSCH.

In the rest of this section, we analyze previous and current standardized solutions developed to operate IPv6 over IoT and analyze its applicability with the LPWANs technologies.

\section{A. 6LoWPAN and 6lo}

In late 2004, the Internet Engineering Task force (IETF) created a work group (WG) to enable IPv6 over IEEE802.15.4 networks, it was named as 6LoWPAN. This WG created an adaptation layer [13] that allows constraint devices to transmit/receive data using the Internet protocol IPv6. Table IV presents the specifications of 6LoWPAN adaptation layer. This project was completed and closed at the end of 2012. Following the growth of communication technologies in the field of Iot with constrained devices, IETF decided to extend the project in order to support a new generation of wired and wireless technologies with IPv6. In 2013, a WG has been formed to run IPv6 over Networks with ResourceConstrained Node (6lo) [14]. With the same concepts, 6lo extends 6loWPAN and supports IPv6 over new technologies. Table IV presents the specifications of 6Lo.

LPWANs technologies are even more constrained than 6LoWPAN and 6lo support [15]. Due to:

- Lack of layer 2 fragmentation: These solutions requires fragmentation of data over several packets on L2 (Data link) layer in case data not fill in one payload frame.
Whereas, most of LPWANs technologies do not support fragmentation at L2 level.

- Message rate limit, due to region regulations: An average of 30 seconds uplink time on air, per day, per device.

- Asymmetry and unsynchronized UL/DL: Most of LPWANs technologies are an UL (uplink) technologies. DL (downlink) rarely used to configure the ED or to return an acknowledgment for an UL. For that UL/DL communication in these technologies is asymmetry. Moreover, no synchronization requires to avoid complexity, consuming the time on air allowed per day, and to reserve the battery.

- Bit rate and Maximum Transmitted Unit (MTU) size is lower than what considered in those technologies 6LoWPAN and 6lo.

- Maximum header compression of IPv6/UDP supported is 6 bytes which corresponds to $10 \%$ of the frame payload on LPWANs and 37 bytes if global addresses is used (62\% of the frame payload).

For that, 6LoWPAN and 6lo solutions cannot be used in LPWANs technologies.

\section{B. $6 \mathrm{TiSCH}$}

In November 2013, a new WG in IETF was formed to develop a standard that allows IPv6 to pass through TimeSlotted channel Hopping (TSCH) mode of IEEE802.15.4e datalinks [16]. 6TiSCH [16] vision was expected to be a standard for low-power wireless industrial monitoring applications. 6TiSCH was a solution for mesh networks that use a deterministic slotted channels. These time slotted channels avoid collisions, reduce power consumption and enable the balancing of data over the channel. 6TiSCH specifications are presented in the Table IV. Even though, 6TiSCH solution can be adopted to LPWANs technologies but it is still not applicable, because their is no synchronization or Time slotted neither the need for scheduling in LPWANs. Most of the LPWANs are star topology not mesh. For that, 6TiSCH could not be used as a solution for LPWANs technologies.

\section{C. $\mathrm{ROHC}$}

The RObust Header Compression (ROHC) [17] protocol was standardized by IETF in 2012 for multimedia data flows in a point to point channel. It is a statefull compression scheme proposed to compress the headers to reduce bandwidth usage for limited capacity or costly networks, such as satellite links and cellular networks. ROHC defines a lossless compression scheme that only compresses the headers without approaching the network packet. ROHC compression scheme uses three levels, where each have its own header format. ROHC sends 52 bytes of header in first level, then 15 to 34 bytes in second, and 2 to 7 bytes in the third level.ROHC compression scheme is able to compress IPv6/IPv4, UDP, UDP-Lite, RTP (Real-time Transport Protocol) and TCP headers. This protocol is efficient in compressing VoIP streams that use RTP as transport protocol. ROHC specifications are presented in Table IV. Although ROHC provides the required level of header compression, ROHC solution is not applicable to LPWANs 
networks, because there is neither streams or continuous data transmission in such technologies nor synchronization. Also, ROHC implementation is complex. It requires significant amount of memory and processing time with respect to the devices constraints. ROHC solution is adapted to multimedia and streaming data with high data rate and does not take into consideration the constraints on processing and energy. Moreover, the bandwidth is extremely short to send full headers in LPWANs and there is no routing or bidirectional (full duplex) data at same time in such technologies.

\section{IPHC and NHC}

LOWPAN IPHC and LOWPAN NHC are another IP header compression solutions defined in RFC 6282 [18]. These solutions are the results of the non applicability of $\mathrm{HC} 1$ and HC2 [19] solutions in 6LoWPAN. IPHC is a hybrid compression scheme that implements a stateless compression scheme with the link-local address, and a statefull compression scheme used to compress global and multi-cast addresses. In best case scenario, IPHC solution can compress IPv6 header using link-local communication down to two bytes, and three bytes using global and multi-cast communication. Beside IPHC, NHC supports compression of the next header. In best case scenario, NHC can compress the UDP header down to one byte, but in practical the compression size is down to five bytes [20]. LOWPAN_IPHC specifications are presented in Table IV. Most of the LPWANs technologies are highly constrained networks that have limited frame sizes. The HC1/HC2 or IPHC/NHC solutions are still not able to achieve the required level of compression. Also, these schemes do not consider the compression of application layer headers. This requires a new scheme to be executed beside IPHC to compress the application layer headers.

\section{E. ROLL and RPL}

In 2012, the Routing over Low power Lossy networks (ROLL) WG at IETF released the protocol IPv6 Routing Protocol for Low-power and lossy networks (RPL for LLNs). LLNs consist of massive number of constrained devices that are battery powered with low processing distributed in a wide geographical area. These nodes are interconnected with lossy links that are recognized as low data rates links. This type of networks also supports point-to-multipoint and multipoint-topoint communications. RPL [21] is a distance vector routing protocol designed for LLNs that uses IPv6. This protocol avoids routing loops when the network topology is modified. RPL specifications are presented in Table IV. Most of LPWANs technologies are based on star topology. Therefore, so far we can consider that routing issues are not recurrent in LPWANs technologies.

\section{F. CORE and CoAP}

In 2014, the Constrained RESTful Environments (CORE) WG at IETF issued the Constrained Application Protocol (CoAP). CoAP is a RESTful framework specified for constrained nodes that runs on constrained IP networks. It is based on asynchronous request/response communication between the applications running on the devices. For simplified integration on the web, this service layer protocol was designed to be translated easily into HTTP. This protocol also supports machine-to-machine (M2M) communication using multicast with small overheads. CoAP is a light application layer protocol with tiny headers and small size message that runs over UDP protocol. Also, it is very suitable for low-memory and low-power devices. Operating IPv6 over CoAP can lead to a potential convergence layer in LPWAN. This layer provides the application executed on the device with seamless connectivity with underlying radio technology. CoAP specifications are presented in Table IV. Currently, the WG of CORE is using the Structure identifiers (SID) to reduce the payload size. This method solved the data limitation constraint in LPWAN technologies, where the headers are reduced by creating a dictionary used to match URL into small identity. Moreover, CoAP as a standard does not consider the requirement of mobility in IoT network environment [22], but this is not a limitation and many solutions have been proposed in this domain.

\section{G. SCHC}

The Static Context Header Compression (SCHC) [23] is a header compression scheme that supports fragmentation level, and is specially tailored for LPWANs technologies. SCHC was designed for constrained networks with low bandwidth and IoT standards that does not support L2 layer fragmentation. SCHC compression techniques offer flexibility when dealing with headers. In the last release (December 2017), LPWAN WG at IETF described the SCHC compression/decompression framework and applied it to IPv6/UDP as well as CoAP. However, SCHC solution is not limited to LPWANs, it could be extended to include other protocol stacks. The work on this solution is still on progress, but $\mathrm{SCHC}$ is now in a mature level. SCHC compression scheme considers that there is a common static context saved on each ED and this context does not change during packet transmission. SCHC specifications are presented in Table IV. SCHC avoids the complexity of synchronization mechanisms which make it a good solution for LPWANs. Most of LPWAN technologies protocol stack does not support fragmentation when data does not fit in one frame. This problem can be solved by adopting the fragmentation mechanism supported by SCHC. This solution provided by SCHC can be applicable on most LPWANs technologies without modifying the protocol stack. It is executed at an upper level to compress the IPv6/UDP headers into Rule ID and forward the data to lower layers. If the data does not fit in one L2 frame, fragmentation mechanism is applied.

\section{CONCLUSIONS}

This paper provides a brief summary of the three low power wide area networks technologies: LoRaWAN, DASH7 Alliance, and Narrow Band-IoT. The three technologies have similar architectures. The architecture components and their specifications have been described. Then, we provided an 
TABLE IV

PROTOCOLS SPECIFICATIONS

\begin{tabular}{|c|c|c|c|c|c|c|c|c|}
\hline \multirow{2}{*}{ Specification } & \multicolumn{8}{|c|}{ Technology Support } \\
\hline & 6LoWPAN & 6Lo & $6 \mathrm{TiSCH}$ & ROHC & IPHC & RPL & CoAP & SCHC \\
\hline Standard & IEEE 2012 & \multicolumn{2}{|c|}{ IEEE start in 2013} & IEEE 2012 & IEEE 2011 & IEEE 2015 & IEEE 2014 & IEEE start in 2016 \\
\hline In progress & No & Yes & \multicolumn{5}{|c|}{ No } & Yes \\
\hline $\begin{array}{l}\text { Data Rate } \\
\text { (in kbps) }\end{array}$ & $\begin{array}{c}20 / 40 / \\
250\end{array}$ & $\begin{array}{c}9.6 / 40 / \\
100\end{array}$ & \multirow{4}{*}{$\begin{array}{l}\text { According } \\
\text { to 6LoWPAN }\end{array}$} & \multicolumn{4}{|c|}{$\begin{array}{l}\text { based on } \\
\text { MAC/PHY }\end{array}$} & $0.5-250$ \\
\hline $\begin{array}{l}\text { Identity } \\
\text { header size }\end{array}$ & \multicolumn{2}{|c|}{$2-8$ bytes } & & $\begin{array}{l}70 \% \text { header } \\
\text { compression }\end{array}$ & $\begin{array}{c}2-3 \\
\text { bytes }\end{array}$ & $\begin{array}{l}\text { Based on used } \\
\text { Encapsulated } \\
\text { protocol }\end{array}$ & \multirow{2}{*}{$\begin{array}{l}4 \text { bytes }+ \\
\text { technology } \\
\text { headers }\end{array}$} & First Byte \\
\hline $\begin{array}{l}\text { Maximum L2 } \\
\text { payload size } \\
\text { (in bytes) }\end{array}$ & 105 & 158 & & $15-20$ & \multicolumn{2}{|c|}{$\begin{array}{l}\text { based on } \\
\text { MAC/PHY }\end{array}$} & & $\begin{array}{l}\text { based on } \\
\text { MAC/PHY }\end{array}$ \\
\hline fragmentation & \multicolumn{2}{|c|}{ Yes } & & Yes & \multicolumn{3}{|c|}{ No } & Yes \\
\hline Protocol stack & \multicolumn{2}{|c|}{$\begin{array}{c}\text { Adaptation layer } \\
\text { above } \\
\text { Link layer } \\
\end{array}$} & $\begin{array}{c}\text { Between } \\
\text { 6LoWPAN \& } \\
\text { Link layer }\end{array}$ & \multicolumn{2}{|c|}{$\begin{array}{l}\text { Application } \\
\text { layer }\end{array}$} & $\begin{array}{c}\text { Routing } \\
\text { Protocol over } \\
\text { Network layer } \\
\end{array}$ & $\begin{array}{l}\text { Application } \\
\text { layer }\end{array}$ & $\begin{array}{l}\text { adaptation layer } \\
\text { below IPv6 \& } \\
\text { above Link layer }\end{array}$ \\
\hline Mobility & \multicolumn{2}{|c|}{ Yes } & Use RPL & \multicolumn{2}{|c|}{$\begin{array}{l}\text { depend on used } \\
\text { routing Protocol }\end{array}$} & Yes & \multicolumn{2}{|r|}{ No } \\
\hline Synchronous & \multicolumn{3}{|c|}{ Yes } & - & Yes & - & \multicolumn{2}{|r|}{ No } \\
\hline Communication & \multicolumn{3}{|c|}{ Symmetrical and Asymmetrical } & Symmetrical & - & Sym. and Asym. & \multicolumn{2}{|c|}{ Asymmetrical } \\
\hline $\begin{array}{l}\text { Applicable } \\
\text { with LPWANs }\end{array}$ & \multicolumn{6}{|c|}{ No } & \multicolumn{2}{|r|}{ Yes } \\
\hline Topology & \multicolumn{3}{|c|}{ Star and Mesh } & \multicolumn{2}{|c|}{$\begin{array}{l}\text { Based on, } \\
\text { MAC/PHY }\end{array}$} & Mesh & $\begin{array}{l}\text { Based on, } \\
\text { MAC/PHY }\end{array}$ & Star \\
\hline
\end{tabular}

analysis of the standardized solutions used to run IP over LPWANs technologies. Finally, we concluded that the CoAP architecture and SCHC scheme provide the best approach that can be adopted as base solution for LPWANs technologies. Future works will consider the mobility management within these solutions to provide mobility for ED between different standards under heterogeneous technologies.

\section{REFERENCES}

[1] "Gartner says 6.4 billion connected things will be in use in 2016 , up 30 percent from 2015," https://www.gartner.com/newsroom/id/3165317, November 10, 2015.

[2] P. Wu, Y. Cui, J. Wu, J. Liu, and C. Metz, "Transition from ipv4 to ipv6: A state-of-the-art survey," IEEE Communications Surveys Tutorials, vol. 15 , no. 3 , pp. 1407-1424, Third 2013.

[3] "Google ipv6 stats on february $20 \quad 2018$ at https://www.google.com/intl/en/ipv6/statistics.html," 2018.

[4] W. Kastner, M. Kofler, M. Jung, G. Gridling, and J. Weidinger, "Building automation systems integration into the internet of things the iot6 approach, its realization and validation," in Proceedings of the 2014 IEEE Emerging Technology and Factory Automation (ETFA), Sept 2014, pp. 1-9.

[5] S. Ziegler, A. Skarmeta, P. Kirstein, and L. Ladid, "Evaluation and recommendations on ipv6 for the internet of things," in 2015 IEEE 2nd World Forum on Internet of Things (WF-IoT), Dec 2015, pp. 548-552.

[6] S. Ziegler, C. Crettaz, and I. Thomas, "Ipv6 as a global addressing scheme and integrator for the internet of things and the cloud," in 2014 28th International Conference on Advanced Information Networking and Applications Workshops, May 2014, pp. 797-802.

[7] LoRaWAN Specification, 2016th ed., LoRa Alliance, https://www.loraalliance.org/lorawan-for-developers.

[8] F. Sforza, "Communications system," Mar. 26 2013, uS Patent 8,406,275, https://www.google.com/patents/US8406275. [Online]. Available: https://www.google.com/patents/US8406275

[9] "Semtech homepage, http://www.semtech.com/," http://www.semtech.com/, 2017.

[10] M. Weyn, G. Ergeerts, L. Wante, C. Vercauteren, and P. Hellinckx, "Survey of the dash7 alliance protocol for $433 \mathrm{mhz}$ wireless sensor communication," International Journal of Distributed Sensor Networks, vol. 9, no. 12, p. 870430, 2013. [Online]. Available: https://doi.org/10.1155/2013/870430
[11] DASH7 Alliance Mode Specification, 2017th ed., DASH7 Alliance Std. "http://www.dash7-alliance.org/dash7-alliance-protocol-specificationv1-1-ready-for-download/",

[12] Cellular system support for ultra-low complexity and low throughput Internet of Things (CIoT) (Release 13), 2015th ed., 3rd Generation Partnership Project (3gpp), http://www.3gpp.org/ftp/Specs/archive/45_series/45.820/.

[13] Z. Sheng, S. Yang, Y. Yu, A. V. Vasilakos, J. A. Mccann, and K. K Leung, "A survey on the ietf protocol suite for the internet of things: standards, challenges, and opportunities," IEEE Wireless Communications, vol. 20, no. 6, pp. 91-98, December 2013.

[14] I. Farris, S. Pizzi, M. Merenda, A. Molinaro, R. Carotenuto, and A. Iera "6lo-rfid: A framework for full integration of smart uhf rfid tags into the internet of things," IEEE Network, vol. 31, no. 5, pp. 66-73, 2017.

[15] C. Gomez, J. Paradells, C. Bormann, and J. Crowcroft, "From 6lowpan to 6lo: Expanding the universe of ipv6-supported technologies for the internet of things," IEEE Communications Magazine, vol. 55, no. 12 pp. 148-155, DECEMBER 2017.

[16] D. Dujovne, T. Watteyne, X. Vilajosana, and P. Thubert, "6tisch deterministic ip-enabled industrial internet (of things)," IEEE Соттиnications Magazine, vol. 52, no. 12, pp. 36-41, December 2014.

[17] "Ieee standard for wirelessman-advanced air interface for broadband wireless access systems," IEEE Std 802.16.1-2012, pp. 1-1090, Sept 2012.

[18] J. Hui and P. Thubert, "Rfc 6282 compression format for ipv6 datagrams over ieee 802.15.4-based networks." rFC 6282 in (Sept. 2011). Last updated Sept. 2016.

[19] J. H. G. Montenegro, "Transmission of ipv6 packets over ieee 802.15.4 networks." rFC 4944. (Sept. 2007).

[20] K. Q. Abdelfadeel, V. Cionca, and D. Pesch, "Lschc: Layered static context header compression for lpwans," in Proceedings of the 12th Workshop on Challenged Networks, ser. CHANTS '17. New York, NY, USA: ACM, 2017, pp. 13-18. [Online]. Available: http://doi.acm.org/10.1145/3124087.3124092

[21] J. Ko, A. Terzis, S. Dawson-Haggerty, D. E. Culler, J. W. Hui, and P. Levis, "Connecting low-power and lossy networks to the internet," IEEE Communications Magazine, vol. 49, no. 4, pp. 96-101, April 2011.

[22] S. M. Chun and J. T. Park, "Mobile coap for iot mobility management," in 2015 12th Annual IEEE Consumer Communications and Networking Conference (CCNC), Jan 2015, pp. 283-289.

[23] "Lpwan static context header compression (schc) and fragmentation for ipv6 and udp," https://tools.ietf.org/html/draft-ietf-lpwan-ipv6-staticcontext-hc-09, Dec. 22, 2017. 